\title{
Relationship Between Tetanus Antitoxin Titration Level and Vaccination History
}

Meltem Işıkgöz Taşbakan ${ }^{1}$, Raika Durusoy ${ }^{2}$, Selma Tosun ${ }^{3}$, Deniz Akyol ${ }^{1}$, Hüsnü Pullukçu,

Tansu Yamazhan ${ }^{1}$ ${ }^{1}$ Department of Infectious Diseases and
Clinical Microbiology, Ege University
Medical Faculty, Izmir
${ }^{2}$ Department of Public Health, Ege
University Medical Faculty, Izmir
${ }^{3}$ Department of Infectious Diseases and
Clinical Microbiology, Bozyaka
Education and Research Hospital, Izmir

\section{Correspondence author:}

M.D Deniz Akyol

Ege University Infection Diseases and Clinical Microbiology

Email: denizakyol416@gmail.com
Received: 27.11.2017,

Accepted: 22.12.2017

DOI: $10.5799 /$ jcei.382398

\section{A B S T R A C T}

Objectives: We aimed to determine tetanus antitoxin levels and to evaluate their relationship with history of vaccination among patients applying to the outpatient clinics of a University hospital.

Methods: A questionnaire including socio-demographic characteristics and tetanus vaccination status was applied and blood samples taken from 218 subjects between 1 and 30 June 2015. Participants were classified into five groups according to their vaccination timing.

Results: The mean age of participants was $46.7 \pm 15.4$ years and 134 (61.5\%) were women. Tetanus antitoxin levels were found weak positive in 54 (24.8\%) patients, positive in 44 (20.2\%) and strong positive in 120 (55.0\%). Tetanus antitoxin level positivity was significantly associated with vaccination timing according to history. Among 105 participants who did not remember being vaccinated or who knew they were vaccinated but did not remember the date, 16 (15.2\%) remembered the vaccination time when their injury, military service and pregnancy were questioned specifically. Antitoxin levels decreased with increasing age independent of gender (0.9-fold increase/year).

Conclusion: We found that the booster dose recommended every 10 years was not applied sufficiently. Tetanus vaccination history must be questioned in more detail among people who do not remember/know their vaccination history, with specific questions regarding pregnancy, military service and injury histories.

Key words: C.tetani, tetanus, vaccine, immunization history

\section{INTRODUCTION}

Tetanus is an acute and usually lethal disease and progresses with uncontrollable muscular cramps caused by tetanospasmin which is a neurotoxin formed by a gram-positive anaerobic bacillus on the lesion called Clostridium tetani. C. tetani is primarily found in the soil and in the intestines of domestic animals and humans [1]. Its route of entry is contaminated lesions. Disease develops if anaerobic conditions appropriate to development of vegetative form of toxin are present [2].

Tetanus is a widespread disease and can be effectively prevented through vaccination with
$100 \%$ efficacy if the required vaccine schedule is completed [3] In our country, the implementation of the tetanus vaccine has started in 1968. According to the current childhood vaccination schedule, three doses of DPT are applied at second, third and fourth months with a booster dose at 16-24 months, followed by Td boosters at 6 and 14 years. As for the adult vaccination schedule, it is regularly applied to pregnant women and women in the age of fertility between 15-49 years to complete five dose schedules (Table 1) [4], while one dose is applied to men performing their military service. Currently there is no other vaccine recommendation or implementation for adults in Turkey. 
National tetanus vaccination programs for adults and specifically populations over the age of 60 are present in many European countries and some of them are presented in Table 2.

Today although newborn tetanus is not observed any more through the "Maternal Neonatal Elimination Program" being carried out in our country, tetanus cases can still be encountered in adulthood and especially in advanced ages. In a study carried out in England between 2001 and 2014 where up-to-date epidemiologic tetanus data were shared, the mortality related to tetanus was found to be $11 \%$. It was determined that all the cases were over 45 years and their tetanus immunization was not completed [5].

Except pregnant women and soldiers, tetanus vaccination and when necessary immunoglobulin application is generally carried out following an injury. Since permanent immunity is not established following tetanus disease, vaccination should not be neglected in those individuals recovered from this disease. For adults who were not vaccinated or who do not remember their vaccination, the primary vaccination is three doses. Two doses are applied with four-week intervals and the third dose tetanus vaccine should be performed 6-12 months after the second dose. The level of antitoxin titers formed against vaccine decreases within years. $5 \mathrm{~A}$ booster shot should be made once every 10 years.

In a study from Denizli, another province of the Aegean Region in Turkey, only 2076 (2.6\%) of the 80,047 individuals $\geq 65$ years were found to be vaccinated against tetanus in the last 10 years according to their family doctors' data in 2011 [6]. In a study carried out in Yozgat province (Central Anatolia) and published in 2016, protective tetanus antitoxin titers were detected among $62.9 \%$ of 267 individuals over 18 years and some differences of protective antibody titers were observed according to education levels, occupations, place of residence and tetanus vaccine application during pregnancy [7]. As antitoxin titers decrease with age, and as no systematic tetanus vaccination is implemented to adults except when injured and to women $>49$ years, and as the limited evidence from Turkey shows a lack of protection against the disease, we aimed to determine tetanus antitoxin levels of patients who applied to the Infectious Diseases polyclinic of our hospital for various reasons except tetanus and to assess the relationship of the antitoxin levels with their vaccination history.

Table 1. Tetanus vaccination schedule of women in the age of fertility (15-49 years) and/or pregnant women in Turkey ${ }^{[4]}$

\begin{tabular}{lll}
\hline Number of doses & Timing of application & Duration of protection \\
\hline Td1 & In the fourth month of pregnancy or first visit & No protection \\
Td2 & At least 4 weeks after Td1 & $1-3$ years \\
Td3 & 6 months after Td2 & 5 years \\
Td4 & At least 1 year after Td3 or in the next pregnancy & 10 years \\
Td5 & At least 1 year after Td4 or in the next pregnancy & During the fertility period \\
\hline
\end{tabular}

Table 2. National vaccination programs against tetanus in different countries of Europe (Countries are listed in alphabetical order)

\begin{tabular}{lll}
\hline Country & Adult (18-60 years) & Elderly (>60 years) \\
\hline Austria & Every 10 years & Every 5 years \\
\hline Belgium & Every 10 years & No suggestion \\
\hline Croatia & 1 time & 1 time \\
\hline Czech Republic & Every $10-15$ years & No suggestion \\
\hline Denmark & No suggestion & No suggestion \\
\hline Finland & Every 10 years & No suggestion \\
\hline France & Every 20 years & Every 10 years \\
\hline Greece & Every 10 years & No suggestion \\
\hline Italy & Every 10 years & No suggestion \\
\hline Norway & No suggestion & No suggestion \\
\hline Slovakia & Every 15 years & No suggestion \\
\hline Spain & No suggestion & 1 time \\
\hline Sweden & Every 20 years & No suggestion \\
\hline
\end{tabular}

\section{SUBJECTS and METHODS}

This is a cross-sectional study conducted in Ege University located in Izmir, Turkey. Ege University Hospital is the largest tertiary care hospital in Aegean Region and many patients from different provinces of the region are referred to or apply directly to this hospital. A sample size of 222 patients was determined using $50 \%$ prevalence, 0.07 error, $95 \%$ confidence and $10 \%$ nonresponse. Among 21,182 patients over 16 years of age referred to our hospital's central laboratory from all outpatient clinics between 1 and 30 June 2015, the study was explained to patients applying in less busy hours and 218 accepted to participate in the study and completed the informed consent form, with 10-15 patients enrolled per day. Participants completed a questionnaire including 14 questions related to their socio-demographic data and tetanus vaccination history. Those who refused to participate in the study and those under 16 years of age were excluded from study.

In this survey the independent variables including age, gender, job, occupation, tetanus vaccination status and timing, injury history and vaccination following injury, data related to military 
service and pregnancy and vaccination against four other diseases (influenza, pneumonia, hepatitis B and measles) were questioned. According to the four tetanus vaccination questions (tetanus vaccination in general, wound management, military service and pregnancy) participants who remembered the timing of their vaccination were classified into five groups according to vaccination timing; within the last 2 years, within last 3-5 years, 6-10 years ago, more than 10 years ago and those who do not remember/ know. Individuals who knew that they were vaccinated but did not remember the timing formed another group and those who did not remember or did not know their vaccination status were included in another group (those who do not remember/know). Individuals were asked if they had any of the following occupations: construction work, farm work, metalworking, scrap dealing, waste collecting, gardening, forest work, husbandry or work related to fertilizers. Participants declaring any of these were considered as having a risky occupation.

The dependent variable of the research is tetanus antitoxin titer. For tetanus antitoxin evaluation, $10 \mathrm{ml}$ was isolated from the blood sample requested for biochemistry and scrutinized with C.tetani toxin 5S IgG-ELISA kit (NovaLisa-Novatech immundiagnostica, Germany). In line with the recommendations of the manufacturer, serum samples were diluted at a rate of $1 / 100$ and studied with micro EIA(enzyme immunoassay) method. According to the test results, samples below $<0.01 \mathrm{U} / \mathrm{ml}$ were considered negative, those between $0.01-0.5 \mathrm{IU} / \mathrm{ml}$ weak positive, those between $0.51-1.0 \mathrm{IU} / \mathrm{ml}$ positive and those $>1.1 \mathrm{U} / \mathrm{ml}$ strong positive.

The descriptive data were presented with numbers and percentages. The statistical significance level was determined as $\mathrm{p}<0.05$. The chi-square test was used in univariate analyses for categorical variables. For advanced analyses, participants were divided into two groups [antitoxin titer sufficient $(>0.1 \mathrm{IU} / \mathrm{ml})$ and insufficient (0.01-0.1 IU/ml)] in terms of antitoxin titer and multivariate analyses were conducted with logistic regression, adjusting for gender, age and tetanus vaccination history. Age variable was included as continuous variable in the model.

\section{RESULTS}

The study included 218 individuals [ 134 women (61.5\%), 84 males (38.5\%); mean age: $46.7 \pm 15.4$ years]. Participants' sociodemographic characteristics and risky occupations in terms of tetanus are presented in Table 3.

Among 105 participants who replied that they did not remember being vaccinated or who knew they were vaccinated but did not remember the date when their tetanus vaccination status was asked in general, 16 (15.2\%) remembered the vaccination time when their injury, military service and pregnancy histories were questioned specifically. Again, we saw that among 52 individuals who had replied the first general question as vaccinated $>10$ years ago, 7 (13.5\%) remembered being vaccinated 6-10 years ago when these three histories were asked specifically. Similarly, we identified that 4 of (19.0\%) 21 individuals who reported they had been vaccinated in the last 6-10 years had in fact been vaccinated within the last five years. These patients were re-grouped according to their vaccination timing in the specific three questions. Participants' tetanus vaccination history is given in Table 4.

Tetanus antitoxin levels were found weak positive in $54(24.8 \%)$ patients, positive in 44 (20.2\%) and strong positive in 120 (55.0\%). The association these tetanus antitoxin levels with gender, vaccine history and risky work are explored in Table 5.

Vaccination history, independent of age and gender, was related to sufficiency of tetanus antitoxin levels. The sufficiency of antitoxin levels in participants vaccinated within the last 1-2 years were 5.2 times more than those did not remember/know and 4.7 times more than those vaccinated within the last 5-10 years. Besides, there was a 0.91 -fold reduction in antitoxin sufficiency with each one-year increment in age, independent of gender and vaccination history (Table 6).

Table 3. Participants' sociodemographic characteristics

\begin{tabular}{|c|c|c|c|c|}
\hline Feature & Women $(n=134)$ & Men $(n=84)$ & $p$ & Total \\
\hline Age groups $(n=218)$ & & & 0.003 & \\
\hline $16-49$ years & $88(65.7)$ & $35(42.2)$ & & $123(56.7)$ \\
\hline 65 years and over & $18(13.4)$ & $16(19.3)$ & & 34 (15.7) \\
\hline Job & & & $<0.001$ & \\
\hline Housewife & $62(46.3)$ & $0(0.0)$ & & $62(28.4)$ \\
\hline Student & $8(6.0)$ & $4(4.8)$ & & $12(5.5)$ \\
\hline Health Employee & $13(9.7)$ & $2(2.4)$ & & $15(6.9)$ \\
\hline Other & $26(19.4)$ & $31(36.9)$ & & $57(26.1)$ \\
\hline Unknown & $14(10.4)$ & 16(19.0) & & $30(13.8)$ \\
\hline Risky occupation & & & 0.009 & \\
\hline
\end{tabular}


Table 4. Participants' distribution according to tetanus and other adult vaccination histories

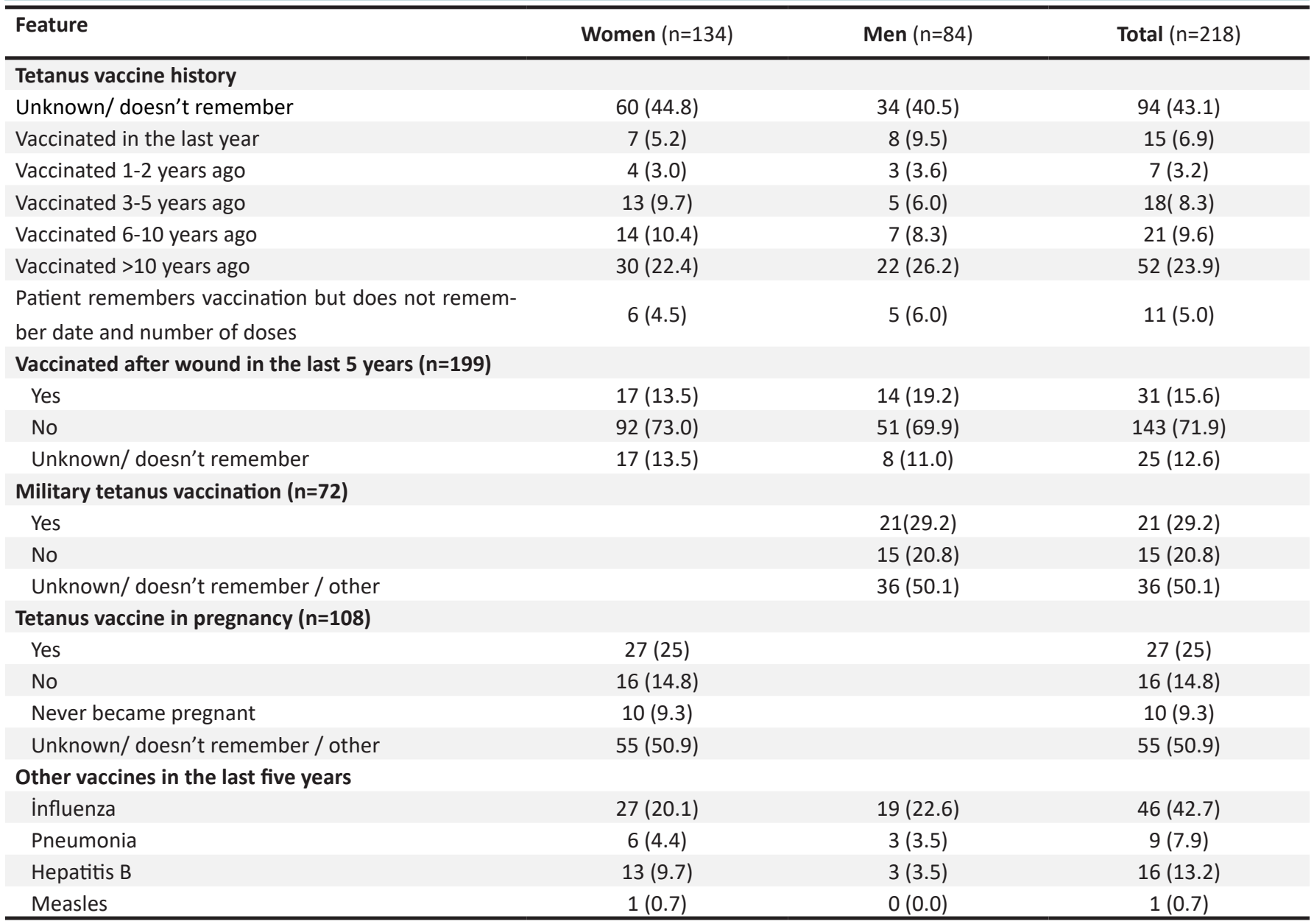

Table 5. Tetanus antitoxin levels according to gender, vaccine history and risky work

\begin{tabular}{|c|c|c|c|c|c|}
\hline & $\begin{array}{l}\text { Weak Positive } \\
(0.01-0.1)\end{array}$ & $\begin{array}{l}\text { Positive } \\
(0.11-0.5)\end{array}$ & $\begin{array}{l}\text { Strong Positive } \\
\text { ( } 0.51 \text { and over) }\end{array}$ & Total & p \\
\hline Gender & & & & & 0.595 \\
\hline Female & $36(26.9)$ & $25(18.7)$ & $73(54.5)$ & 134 & \\
\hline Male & $18(21.4)$ & $19(22.6)$ & $47(56.0)$ & 84 & \\
\hline Age group & & & & & 0.001 \\
\hline $15-49$ years & $13(10.6)$ & $18(14.6)$ & $92(74.8)$ & 123 & \\
\hline $50-64$ years & $17(28.3)$ & $18(30.0)$ & $25(41.7)$ & 60 & \\
\hline 65 years and older & $24(70.6)$ & $8(23.5)$ & $2(5.9)$ & 34 & \\
\hline Tetanus vaccine story & & & & & 0.001 \\
\hline Vaccinated in the last two years & $2(9.1)$ & $2(9.1)$ & $18(81.8)$ & 22 & \\
\hline Vaccinated $3-5$ years ago & $2(8.3)$ & $4(16,7)$ & $18(75)$ & 24 & \\
\hline Vaccinated $6-10$ years ago & $5(16.1)$ & $3(9.7)$ & $23(74.2)$ & 31 & \\
\hline Vaccinated $>10$ years ago & $16(30.8)$ & $10(19.2)$ & $26(50.0)$ & 52 & \\
\hline Unknown/ doesn't remember & $29(32.6)$ & $25(28.1)$ & $35(39.3)$ & 89 & \\
\hline Total & $54(24.8)$ & $44(20.2)$ & $120(55.0)$ & 218 & \\
\hline Risky occupation & & & & & 0.832 \\
\hline Yes & $13(25.0)$ & $9(17.3)$ & $30(57.7)$ & 52 & \\
\hline No & $41(24.7)$ & $35(21.1)$ & $90(54.2)$ & 166 & \\
\hline
\end{tabular}


Table 6. Factors associated with tetanus antitoxin sufficiency classified as $>0.10 \mathrm{IU} / \mathrm{ml}$ (multivariate analysis)

\begin{tabular}{lcc}
\hline Feature & OR (\%95 confidence interval) & $p$ \\
\hline Gender & & \\
Female & $1.76(0.85-3.65)$ & 0.130 \\
Male & & $<0.001$ \\
Age & $0.91(0.89-0.94)$ & 0.012 \\
Tetanus vaccine story & & 0.017 \\
Unknown/ doesn't remember & 1 & 0.061 \\
Vaccinated in the last two years & $5.19(1.34-20.07)$ & 0.004 \\
Vaccinated 3-5 years ago & $2.98(0.95-9.34)$ & 0.163 \\
Vaccinated 6-10 years ago & $4.65(1.63-13.30)$ & $1.80(0.78-4.12)$ \\
Vaccinated $>10$ years ago & & \\
\hline
\end{tabular}

\section{DISCUSSION}

Among the serologic methods used in order to evaluate the antibody response formed against tetanus vaccine, in vivo toxin neutralization test (NT) is considered a golden standard [9]. This test can be used in measurement of lower antitoxin titers, such as $0.001 \mathrm{IU} / \mathrm{mL}[10]$. However, it is a laborious test because it requires live animals and excessive amount of samples [11]. Other serologic tests, indirect hemaglutination test (IHA), counterimmune-electrophoresis (CIE), radio immunoassay (RIA) and enzyme-linked immunosorbent assay (ELISA) [9]. The ELISA test we used in our study is a simple, automatable, practical and rapidly response providing test. This has been found valid compared to the NT test, with differing cut-off values according to the procedure applied [12]. However, when the antitoxin levels are low, the test has difficulties in determining the real antitoxin level due to cross-reactions [13].

In our country vaccination to children is applied through neonatal tetanus elimination program initiated in 1994. Among the adult and elderly tetanus vaccination programs in different European countries, Turkey has similar characteristics to most countries with a recommendation of booster dosing every 10 years in adult age and the elderly. In a study carried out in Çorum province (Central Anatolia) in 2014 where vaccination rates were investigated in pregnant women, it was found that $30 \%$ of pregnant women had never been vaccinated during the gestational period and among the remaining vaccinated pregnant women, the vaccination schedule of $40 \%$ had not been completed [14]. The same study concluded that the reason for not having tetanus vaccine was indolence/negligence (40\%).

In order to evaluate the effectiveness of vaccination serological studies are needed [9]. Vaccination efforts targeting adults started in 1968 in Turkey. Various studies in the world have shown that protective antitoxin levels $(>0.1 \mathrm{IU} / \mathrm{ml})$ against tetanus have not reached the targeted high levels. A study conducted in Spain between 1995 and 1998 has reported that $69.4 \%$ of participants aged 45-79 years did not have protective antitoxin level [15]. In another study conducted in Italy in 2003-2004, a lack of protective tetanus antitoxin levels were found among $56.6 \%$ of participants aged $45-64,73.4 \%$ in the age group $65-75,72.1 \%$ in the age group 76-84 and $82.9 \%$ among participants over 85 years of age, indicating a gradual decline in tetanus antitoxin levels within years [16]. The fact that $80.2 \%$ of the cases of tetanus occurring between 2001 and 2010 in Italy were reported in the population aged 65 years and over and 36 (62\%) of the 58 cases of tetanus reported between 1993 and 2007 in Australia were also over 65 years of age supports this increasing risk with increasing lag of time from last the booster dose [17-18]. Our finding of antitoxin levels decreasing 0.9-fold annually independent of gender also supports this increasing risk with age.

In a study conducted in Mersin province in Turkey in 2005 where tetanus seroprevalence was investigated among women between 15-49 years of age, $72.3 \%$ of women were found to be seropositive. Tetanus toxoid (TT) IgG titer of $0.01 \mathrm{IU} / \mathrm{mL}$ and over was considered seropositive in that study. The tetanus toxoid (TT) IgG titer was found $0.1 \mathrm{IU} / \mathrm{mL}$ and over in only $1.5 \%$ of these seropositive women [19]. In the same study, the seropositivity rate was found higher in women in whom the time elapsed after the last TT dose was between 0 - 10 years. In another study carried out in Manisa in 2010, a neighboring province of Izmir, where tetanus antitoxin titers were investigated among individuals over 40 years of age, antibody levels were found immeasurable in $30.9 \%$ of males and $38.8 \%$ of women [20]. In our study tetanus antitoxin levels were found weak positive in $24.8 \%$, positive in $20.2 \%$ and strong positive in $55.0 \%$ of participants, with no participant having undetectable antitoxin levels. 
Although not statistically significant ( $\mathrm{p}=0.595)$, the level of tetanus antitoxin in men was found to be higher in our study compared to women. Statistically significantly higher antibody levels among men compared to women $(p=0.021)$ was found in a study on 734 adults in Austria and published in 2005. A reason of not finding a statistically significant difference in our study might be the smaller number of participants or the effective conduction of the vaccination program targeting women aged 15-49 years in Turkey. The tetanus vaccination histories questioned in the same study from Austria showed a similar significant correlation with antitoxin titers $(\mathrm{p}<0.001)$ as in our study [21].

By asking more specific questions on tetanus vaccination history, like questioning the women on their tetanus vaccination histories during gestational period and men during military service, we have obtained different results as compared to the general question on tetanus vaccination. This demonstrates the importance of detailed questioning in obtaining data. In a multicenter retrospective study involving 25 centers from our country, adult cases of tetanus were also examined. Among a total of 117 tetanus cases evaluated in this study, 38 (32.5\%) had died. When the vaccination histories of the patients were examined, it was learned that only 8 of them were vaccinated against tetanus in the last 10 years, but none of these vaccinated persons had died [22].

The primary limitation of this study is that a probability sampling scheme could not be performed among the patients referred to our hospital's central laboratory. Therefore, new studies that will reflect epidemiology especially in our region are needed. The lack of a question separately evaluating the vaccination status of women during 15-49 years and their gestational period was another limitation of the study, since almost all women are vaccinated during pregnancy due to strict follow-up of family physicians while participation to the 15-49 year-old program (out of pregnancy) is not obligatory and followed. There is a need for studies similar to the one in Austria, in which the pre-vaccination antibody level for tetanus, diphtheria and pertussis affects the humoral immune response and antibody level after vaccination. 23 In our study, the tetanus antitoxin titers in patients with known vaccination history before tetanus vaccination is one of the limitations in terms of the scope of work.

In conclusion, the antitoxin levels were found remarkably higher among participants who were vaccinated in the past two years and the tetanus antibody levels statistically significantly decreased with age. Although the application of a booster tetanus vaccine every 10 years is recommended within the frame of adult vaccine program in Turkey, it is observed that this recommendation is not practiced sufficiently, especially after the age of 50 years. Particularly for those individuals who do not know the vaccination history a booster dose must be applied [4].

Conflict of Interests: The authors declare that they have no conflict of interest.

Financial Disclosure: No financial support was received.

\section{REFERENCES}

1. Taeusch W, Ballard A. Avery's disease of the newborn. Edition 1998; 7: 516-517.

2. American Academy of Pediatrics. Report of the committee on infectious diseases. 1997 Red Book. 24 ${ }^{\text {th }}$ ed. 1997:518-23.

3. CDC Epidemiology and Prevention of Vaccine-Preventable Diseases. The Pink Book: Course Textbook - 13 ${ }^{\text {th }}$ Edition, 2015.

4. Adult Immunization Guide, 2nd Review. May 2016, İstanbul

5. Collins S, Amirthalingam G, Beeching NJ, et al. Current epidemiology of tetanus in England, 2001-2014. Epidemiol Infect. 2016;18:1-11.

6. Ünal S, Tanrı̈̈ver DM, Taş E, Güner İ, Çetin Ö, Sayar İ. The effects of educating family physicians and getting targets for vaccination on pneumococcal vaccination coverage rates. Flora 2015;20(1):10-15.

7. Kader Ç, Balci, Erbay. Evaluation of tetanus antibody levels in adults in Yozgat, Turkey. Turk J Med Sci. 2016;46:646-50.

8. Centralized information system for infectious diseases (CISID) data.euro. who.int/cisid

9. Atabey N, Gökoğlu M. Antitoxin levels detected against tetanus in different age groups. Microbiology Bull. 1990;24:133-40.

10. Aggerbeck H, Pederson B, Heron I. Simultaneous quantitation of diphteria and tetanus antibodies by double antigen, time resolved fluorescence immunoassay. J Immunol Methods. 1996;190:171-83.

11. Ramshorst V. Titration of diphtheria and tetanus antitoxins in sera of low titer. Bull World Health Org. 1971;45:213-8.

12. Sönmez C, Çöplü N, Esen B. Improvement of home-made ELISA technique for detection of anti-tetanus antibodies. Turkish Microbiol Commun Bull. 2010;40:259-66.

13. William P. Fundamental Immunology. $2^{\text {nd }}$ ed. London: Raven Pres, 1998:331-4.

14. Duman N, Yılmazel G, Başcı A, Koçak D. Frequency of vaccination against tetanus in pregnancies participating in the birth preparation class and factors affecting them. Turk Family Physicians Bull. 2015;19:84-9.

15. Bayas JM, Vilella A, Bertran MJ, et al. Immunogenicity and reactogenicity of the adult tetanus-diphtheria vaccine. How many doses are necessary? Epidemiol Infect. 2001;127:451-60.

16. Filia A, Bella A, von Hunolstein C, et al. Tetanus in Italy 2001-2010: a continuing threat in older adults. Vaccine. 2014;32:639-44.

17. Weinberger B. Adult vaccination against tetanus and diphtheria: the European perspective. Clin Exp Immunol. 2017;187:93-9.

18. Quinn HE, McIntyre PB. Tetanus in the elderly--An important preventable disease in Australia. Vaccine. 2007;25:1304-9.

19. Öner S, Buğdaycı R, Kurt A, Öztürk C, Şaşmaz T. Tetanus seroprevalence -among women 15-49 years old, in Mersin, Turkey. Turkiye Klinikleri J Med Sci-2008;28:839-46.

20. Tosun S, Deveci S, Yücetürk M, Dönmez A, Kaplan Y. Investigation of tetanus antitoxin titer levels in people over forty years of age. Turkiye Klinikleri J Med Sci 2012;32:301-7.

21. Hainza U, Jeneweina B, Ascha E, et al. Insufficient protection for healthy elderly adults by tetanus and TBE vaccines. Vaccine 2005;23:3232-5.

22. Infectious Diseases International Research Initiative (ID-IRI). Tetanus in adults: Results of multicenter ID-IRI study. Eur J Clin Microbiol Infect Dis 2017;25:12-6.

23. Kaml M, Weiskirchner I, Keller M. Booster vaccination in the elderly: Their success depends on the vaccine type applied earlier in life as well as on pre-vaccination antibody titers. Vaccine. 2006;24:6808-11. 